\title{
High-Risk Surgically Resected Pediatric Melanoma and Adjuvant Interferon Therapy
}

\author{
Mwe Mwe Chao, $\mathrm{MD}^{1}{ }^{1}$ Jennifer L. Schwartz, $\mathrm{MD}^{2}{ }^{2}$ Daniel S. Wechsler, MD, PhD, ${ }^{1}$ \\ Courtney D. Thornburg, MD, ${ }^{1}$ Kent A. Griffith, MS, MPH, ${ }^{3}$ and James A. Williams, ${ }^{1}{ }^{1}$
}

\begin{abstract}
Background. Pediatric patients with highrisk surgically resected melanoma are at risk for relapse, yet little is known about these young patients and how they tolerate high-dose interferon therapy. Procedure. We reviewed medical records of patients ( $\leq 18$ years) with high-risk melanoma referred to the University of Michigan Pediatric Hematology-Oncology service between January 1989 and July 2003. Results. Fourteen patients were identified with high-risk resected melanoma. The median age at diagnosis was 8.5 years. The median time to establish diagnosis was 9 months. Primary lesions were diagnosed as unequivocal melanoma, atypical epithelioid melanocytic proliferations, or atypical Spitz tumor with indeterminate malignant potential. Twelve patients had a positive sentinel lymph node (SLN) biopsy or a palpable regional lymph node and underwent regional lymph node dissection (LND). Two patients with unequivocal melanoma with Breslow depth $>4 \mathrm{~mm}$
\end{abstract}

had negative SLN biopsies. Twelve patients received adjuvant high-dose interferon. The following toxicities were observed: constitutional symptoms, gastrointestinal symptoms, depression or neuropsychiatric symptoms, myelosuppression, elevated AST or ALT, hypothyroidism, and hypertension. Grade 3 or 4 toxicities were uncommon with exception of neutropenia, resulting in modification of therapy in one patient. All patients are alive and free of disease at follow-up (median 24.5 months). Conclusions. Invasive melanoma can occur in very young children. Despite early signs of malignancy, there is often a delay in diagnosis. Histologically, diagnosis may be difficult because of overlap with Spitz nevi. Pediatric patients tolerated adjuvant high-dose interferon well and may be less likely than adults to require therapy modification secondary to toxicities. Pediatr Blood Cancer 2005;44:441-448. (c) 2004 Wiley-Liss, Inc.

Key words: high-risk melanoma; interferon alpha; pediatric melanoma

\section{INTRODUCTION}

Pediatric melanoma accounts for $1-3 \%$ of all childhood neoplasms, and only $0.8 \%$ of melanomas occur in individuals under 20 years of age [1,2]. Over the past several decades, however, the incidence of melanoma in the United States has risen dramatically [3]. It is projected that 1 in 50 Americans born in the year 2010 will develop melanoma in their lifetime, a marked increase in lifetime risk compared with 1 in 1,500 Americans born in 1935 [4]. Today, children are more likely to develop melanoma during their lifetime than at any other time in history.

Melanoma may occur in previously healthy children or in children with an underlying immunodeficiency or genetic disorder [5-7]. Melanoma may develop in congenital or acquired nevi or arise as a de novo lesion [5,6,8]. As in adult patients, advanced stage at diagnosis confers a poor prognosis [5,7,9-11]. For loco-regional disease, prognosis depends on the stage of disease based on thickness, ulceration status of the primary lesion, and the number and extent (microscopic vs. macroscopic disease) of involved regional lymph nodes [12]. Patients, including children, with either thick localized disease ( $>4 \mathrm{~mm}$ Breslow depth) or with regional lymph node disease (American Joint Committee on Cancer, AJCC stage IIB/C or III, respec- tively) are at high risk for disease recurrence [13]. This has led to the use of adjuvant therapies to complement surgical treatment in an effort to improve disease-free and overall survival in this group.

The mainstay of melanoma treatment in both adults and children is surgical, including a wide local excision (WLE) of the primary site and a lymph node dissection (LND) in the setting of a positive sentinel lymph node (SLN) biopsy or positive tissue diagnosis of a palpable regional lymph node [14-21]. In the past, adjuvant

\footnotetext{
${ }^{1}$ Department of Pediatric Hematology-Oncology, University of Michigan Health System, Ann Arbor, Michigan

${ }^{2}$ Department of Dermatology, University of Michigan Health System, Ann Arbor, Michigan

${ }^{3}$ Department of Biostatistics, University of Michigan Health System, Ann Arbor, Michigan

This study was reported as a poster at the 2003 ASPHO meeting in Seattle, Washington.

*Correspondence to: Dr. Mwe Mwe Chao, Department of Pediatrics, Division of Pediatric Hematology-Oncology, University of Michigan Health System, 1500 East Medical Center Drive, CCGC 4410, Ann Arbor, MI 48109-0936. E-mail: mmchao@umich.edu
}

Received 22 April 2004; Accepted 22 June 2004 
therapy for high-risk resected disease included immunotherapy, vaccines, chemotherapy, and combinations thereof [22-29]. To date, high-dose interferon alfa-2b (HDI) administered initially at 20 million IU $/ \mathrm{m}^{2} /$ day IV 5 times per week for 4 weeks, followed by 10 million IU/ $\mathrm{m}^{2} /$ day SC 3 times per week for 48 weeks has been reputed to be effective [24]. Although there is some disagreement over its benefit, HDI is FDA-approved for high-risk surgically resected disease, and may be considered as the therapy to which all prospective adjuvant treatments should be compared. Interferon alfa- $2 b$ (IFN) is a human recombinant cytokine with antitumor properties and acts by direct antiproliferative and indirect immunomodulary mechanisms [30].

There are several randomized trials of IFN therapy for melanoma using different dosages, modes of administration, and patient populations. Only HDI has been shown to improve relapse-free and possibly overall survival in patients with high-risk resected melanoma [24-26]. These encouraging results led to the use of this regimen to treat children with high-risk melanoma after surgical resection. Unfortunately, the administration of HDI to adults is associated with significant adverse reactions. Based on data from Eastern Cooperative Oncology Group (ECOG) melanoma trials, HDI toxicities included constitutional symptoms, gastrointestinal symptoms, myelosuppression, elevated hepatic enzyme levels, and depression or neuropsychiatric symptoms [24]. The hematologic and hepatic toxicities were reversible on interruption or dose reduction of IFN treatment; however, the constitutional and neurologic toxicities were often of sufficient severity to warrant discontinuation of the medication [24]. This study examines the clinical characteristics of pediatric patients with locally invasive or regionally metastatic melanoma and investigates how these patients tolerated HDI therapy.

\section{METHODS}

The study was approved by the University of Michigan Medical School Institutional Review Board. A retrospective review of medical records was performed on all children with invasive melanoma who presented to the University of Michigan Pediatric Hematology-Oncology clinic in conjunction with the Multidisciplinary Melanoma clinic between January 1989 and July 2003. Patients with high-risk surgically resected melanoma (AJCC stage IIB/C or III) were considered for adjuvant therapy. Individuals electing to be treated with adjuvant IFN received the FDA-approved HDI regimen (20 million IU/ $\mathrm{m}^{2} /$ day IV 5 times per week for 4 weeks followed by 10 million $\mathrm{IU} / \mathrm{m}^{2} /$ day SC 3 times per week for 48 weeks, INTRON A, recombinant interferon alfa-2b; Schering Plough, Kenilworth, NJ). For purposes of the study, the following information was entered into a de-identified research record for each patient: age, race, gender, primary site, presenting signs or symptoms, interval between sign or symptom onset and diagnosis, risk factors, histologic subtype, Breslow depth, surgical treatment, SLN biopsy status, and side effects experienced by patients on HDI therapy graded according to the National Cancer Institute common toxicity criteria [31]. Duration of follow-up was defined in months from the date of diagnosis through July 2003.

The SLN biopsy technique used preoperative intradermal injection of ${ }^{99 \mathrm{~m}} \mathrm{Tc}$-sulfur colloid (1-3 mCi; CIS-US, Inc., Bedford, MA) and intraoperative intradermal injection of isosulfan blue dye (Lymphazurin 1\%; Hirsch Industries, Inc., Richmond, VA) around the intact tumor or biopsy site. The SLN(s) was identified with the aid of a hand-held gamma counter and uptake of blue dye. SLNs were evaluated after serial sectioning and hematoxylin and eosin (H\&E) plus immunohistochemical staining. All histopathology (primary lesions, SLNs, and LND specimens) was reviewed by University of Michigan melanoma dermatopathologists.

Published adult data from ECOG melanoma trials was used to compare adverse events between pediatric and adult patients $[24,32]$. The equality of the proportion of patients experiencing adverse events while undergoing treatment for melanoma was assessed using Fisher's exact test. Two-sided $P$-values $\leq 0.05$ were considered statistically significant.

\section{RESULTS}

Table I reviews the clinical characteristics of pediatric patients with invasive melanoma evaluated at the University of Michigan Pediatric Hematology-Oncology clinic. Two patients had AJCC stage IIB/C disease and 12 had stage III melanoma. There were six males and eight females with a median age at diagnosis of 8.5 years (range 5-18 years). Twelve were Caucasian and two were Hispanic. Primary tumor sites included head and neck $(\mathrm{n}=6)$, trunk $(\mathrm{n}=3)$, and extremity $(\mathrm{n}=5)$ (Fig. 1). Nine (64\%) lesions developed de novo, one (7\%) occurred in a preexisting lesion, and four $(29 \%)$ arose in congenital nevi. The primary lesions were asymptomatic in three patients and were biopsied due to suspicious appearance identified by the primary care physician. Eleven $(79 \%)$ patients noted growth, change in color, itching, ulceration, or bleeding of their lesion prompting an evaluation. The median delay between sign or symptom onset and diagnosis was 9 months. Risk factors for the development of melanoma included immunosuppression secondary to chemotherapy for medulloblastoma $(\mathrm{n}=1)$, personal and/or family history of melanoma $(n=3)$, and history of sunburns $(\mathrm{n}=3)$. Interestingly, one patient was diagnosed with two primary lesions. At 4 years of age this patient developed a lesion on her upper back that was histologically an atypical melanocytic proliferation with indeterminate biologic 


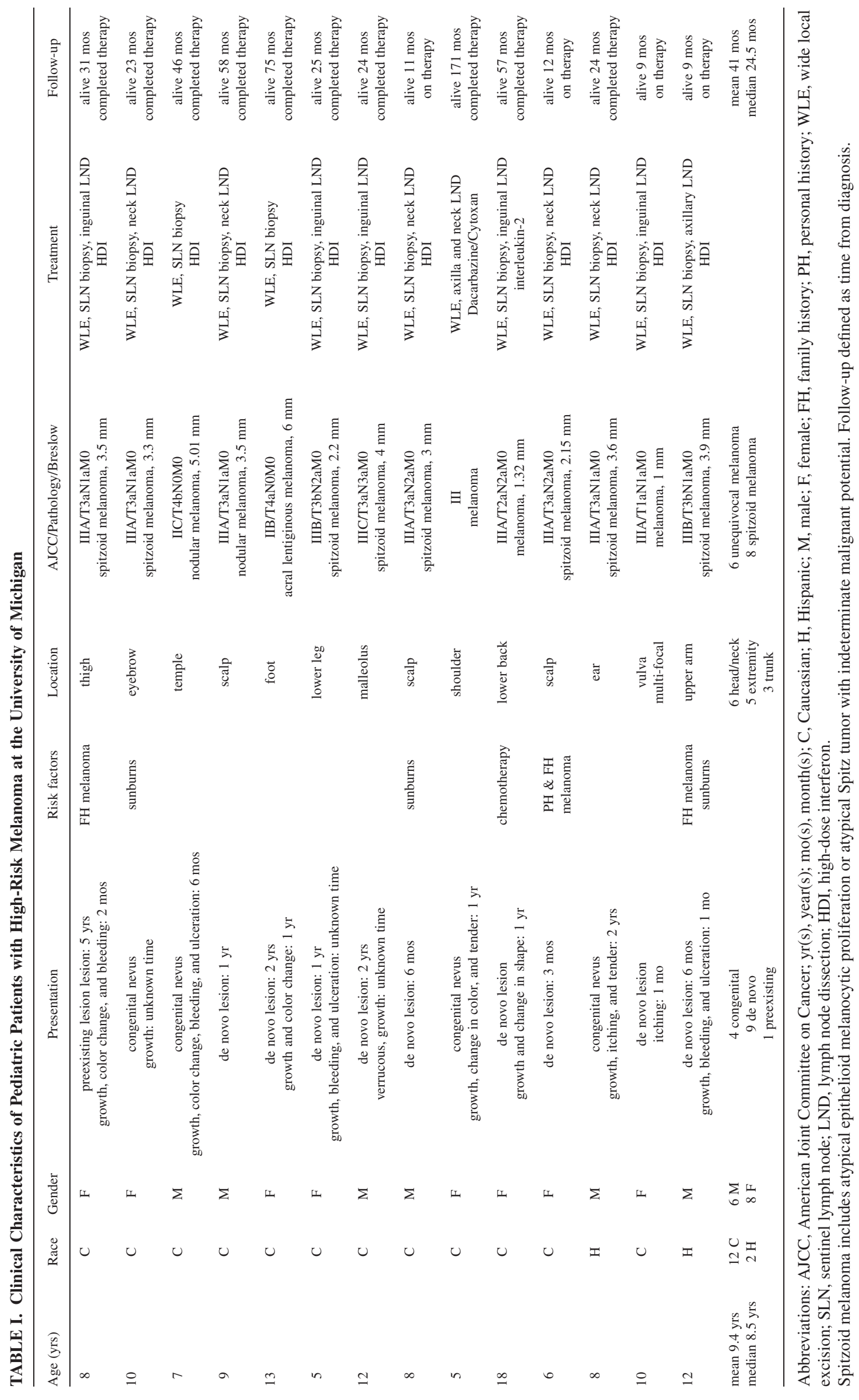



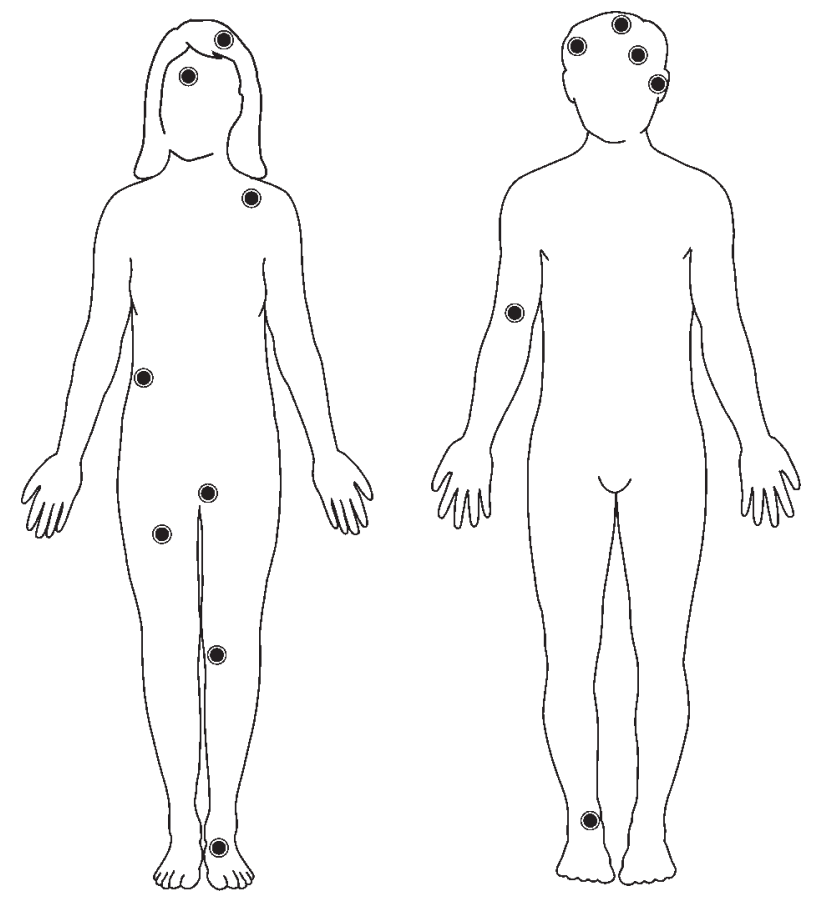

Fig. 1. Primary sites of melanoma. In female patients, melanoma lesions involved all areas of the body. In contrast, the majority of lesions in male patients were localized in the head and neck region.

potential (Breslow depth $1.65 \mathrm{~mm}$ ). The lesion was managed conservatively as a melanoma with surgical resection. Two years later, the patient developed a de novo lesion in her scalp, which was shown to be an atypical epithelioid melanocytic proliferation with metastasis to two regional lymph nodes. None of the patients with a family history of melanoma had documented familial melanoma syndrome or atypical nevus syndrome.

The primary lesion was diagnosed histologically as unequivocal melanoma in six patients and as atypical epithelioid melanocytic proliferation or atypical Spitz tumor with indeterminate malignant potential in eight cases. In the latter group, the primary lesion was reclassified as a spitzoid melanoma following a positive SLN biopsy for metastatic melanoma. Breslow thickness ranged from 1.0 to $6.0 \mathrm{~mm}$ (median $3.5 \mathrm{~mm}$ ). There was evidence of regional lymph node disease on physical examination in one patient. None of the patients had evidence of distant metastases based on history, physical examination, or laboratory or imaging evaluations performed at diagnosis. All patients underwent WLE. SLN biopsy was performed in all patients with clinically negative lymph nodes (13/ 14). Eleven patients had a positive SLN biopsy and underwent regional LND, as did the patient with palpable nodal disease. SLN biopsies were negative in two patients, both with unequivocal melanoma $>4 \mathrm{~mm}$ Breslow thickness. Only microscopic disease was detected in individuals with positive lymph node(s).
TABLE II. Adverse Events in Pediatric Patients Treated With High-Dose Interferon

\begin{tabular}{|c|c|c|}
\hline Adverse event & $\begin{array}{c}\text { All grades } \\
\text { [n }(\%)]\end{array}$ & $\begin{array}{c}\text { Grades } 3 / 4 \\
{[\mathrm{n}(\%)]}\end{array}$ \\
\hline Constitutional/systemic & $11(100)$ & $1(9)$ \\
\hline Fatigue & $7(64)$ & 0 \\
\hline Fever & $10(91)$ & $1(9)$ \\
\hline Myalgia & $6(55)$ & 0 \\
\hline Gastrointestinal & $8(73)$ & $1(9)$ \\
\hline Anorexia & $3(27)$ & 0 \\
\hline Diarrhea/constipation & $4(36)$ & 0 \\
\hline Nausea & $5(45)$ & $1(9)$ \\
\hline Vomiting & $2(18)$ & 0 \\
\hline Weight loss & $6(55)$ & 0 \\
\hline Myelosuppresssion & $11(100)$ & $7(64)$ \\
\hline Neutropenia & $11(100)$ & $7(64)$ \\
\hline Anemia & $3(27)$ & 0 \\
\hline Thrombocytopenia & $1(9)$ & 0 \\
\hline Serious infections ${ }^{\mathrm{a}}$ & 0 & 0 \\
\hline Hepatic & $10(91)$ & $1(9)$ \\
\hline Elevated AST/ALT & $10(91)$ & $1(9)$ \\
\hline Elevated bilirubin & 0 & 0 \\
\hline Hypothyroidism & $2(18)$ & 0 \\
\hline Depression/neuropsychiatric ${ }^{b}$ & $4(36)$ & $1(9)$ \\
\hline Hypertension & $1(9)$ & 0 \\
\hline
\end{tabular}

${ }^{a}$ Serious infections defined as infections requiring intravenous antibiotics or hospitalizations.

${ }^{\mathrm{b}}$ Neuropsychiatric symptoms include irritability and anxiety.

Eleven patients (two AJCC stage IIB/C and nine stage III) received adjuvant HDI at the University of Michigan. The remaining three patients received chemotherapy, other immunotherapy, or HDI at another institution. Seven patients completed HDI without dose adjustment and four patients are currently being treated. In this latter group, one patient experienced neutropenia with an absolute neutrophil count (ANC) of $400 / \mathrm{mm}^{3}$ (grade 4) during the fourth week of the induction phase, and the IFN dose was reduced by $50 \%$ for the remainder of the induction phase without a change in ANC. Therapy was subsequently discontinued for 1 week, and then restarted at $75 \%$ maintenance phase dosage. The other three patients have not required dose adjustments to date.

A summary of toxicities and comparison of adverse events with adult patients are detailed in Table II. Flu-like symptoms including fatigue, fever, or myalgia were reported in $100 \%$ of patients, but in only one patient was this graded 3 or 4 . Fatigue (64\%) and fever $(91 \%)$ occurred temporally in association with IFN infusion and improved with continued therapy. One patient experienced a highgrade fever of $105^{\circ} \mathrm{F}$ without sequelae (grade 3). Mild gastrointestinal symptoms were reported in $73 \%$ of patients and included anorexia (27\%), diarrhea or constipation $(9 \%)$, nausea (45\%), vomiting (55\%), and weight loss (64\%). One patient experienced grade 3 nausea resulting in dehydration and treatment with intravenous fluids. Seven patients experienced weight loss (mean $2.9 \mathrm{~kg}$ ) that 
was regained by the end of therapy. All patients had some degree of neutropenia, and $64 \%$ had grade 3 or 4 toxicity (ANC $<1,000$ cells $/ \mathrm{mm}^{3}$ ) with a mean ANC of $590 / \mathrm{mm}^{3}$. The most severe neutropenia occurred during the final week of the induction phase of therapy. There were no serious infections requiring intravenous antibiotics or hospitalizations. Three patients had positive streptococcal throat cultures. Anemia (27\%) and thrombocytopenia $(9 \%)$ occurred with less frequency and were not severe. Elevations in AST or ALT occurred in $91 \%$ of patients, but no patients had elevations in bilirubin. Only one patient experienced grade 3 elevations in AST and ALT ( $>250$ IU/ L). This occurred during the last week of induction and there was no dose delay or reduction. Hepatic enzymes normalized during maintenance therapy. Depression, anxiety, or irritability occurred in $36 \%$ of patients, and one patient required anti-depressant medication for grade 3 symptoms. Two patients (18\%) had laboratory studies consistent with hypothyroidism; one case occurred immediately after starting HDI and the second case occurred at the end of therapy. Neither patient required thyroid hormone supplementation. One patient had elevated blood pressures during IFN infusion which improved without intervention.

Statistical analysis shows that the proportions of pediatric patients suffering from fatigue or vomiting (all grades) was significantly lower ( $P$-values 0.0024 and 0.0027 , respectively) than for adult patients undergoing the same treatment. However, there were no statistically significant differences between the proportions of patients (pediatric vs. adult) experiencing adverse events for any of the other categories tabulated (Table III). With regards to IFN dose adjustments, pediatric patients $(9 \%)$ were less likely to require a dose modification than adults (50\%) $(P$-value 0.0101). Follow-up data is available for 14 patients, all of whom are alive and are without evidence of disease with median follow-up of 24.5 months from diagnosis (range 9-171 months).

\section{DISCUSSION}

Historically, pediatric melanoma was thought to occur almost exclusively in the second decade of life, in Caucasian children, and children with predisposing conditions such as giant congenital nevi, atypical nevus syndrome, xeroderma pigmentosum, or immunosuppression $[5,6]$. In this study of pediatric patients with high-risk resected melanoma, 12 of 14 patients were Caucasian and interestingly, 2 patients were Hispanic. The median age at diagnosis was 8.5 years, confirming that melanoma, in particular advanced disease, can occur in the first decade of life. Many children did not have obvious underlying risk factors for melanoma. Although the number of patients in this study is small, the results here and elsewhere raise the question of whether there has been a shift in the population characteristics of pediatric melanoma patients [33]. Increased solar exposure has been implicated as an important factor in the pathogenesis of melanoma. Unlike basal or squamous cell carcinoma in which cumulative sun exposure over time leads to cancer, melanoma risk is increased by intermittent intense exposure resulting in blistering or peeling sunburn [34-36]. The highest melanoma rates are in fair-skinned populations in areas of intense sunlight, most notably Australia [36]. Three (21\%) of the study patients reported a history of severe sunburn, making intense sun exposure the most prevalent modifiable documented risk factor for the development of melanoma in this population.

A large portion of patients (79\%) in this study had a prolonged delay (median 9 months) in diagnosis despite signs of a malignant lesion. Of note, there was a year or longer delay in diagnosis in three patients (29\%). The earliest sign of melanoma is a change in size, shape, or

TABLE III. Comparison of Adverse Events in Pediatric and Adult Patients

\begin{tabular}{|c|c|c|c|c|c|c|}
\hline \multirow[b]{2}{*}{ Adverse event } & \multicolumn{2}{|c|}{ Pediatrics $(\%)$} & \multicolumn{2}{|c|}{ Adults $(\%)$} & \multicolumn{2}{|c|}{$\begin{array}{c}\text { Fisher's exact, } \\
\text { two-sided } P \text {-value }\end{array}$} \\
\hline & All grades & Grades 3/4 & All grades & Grades 3/4 & All grades & Grades 3/4 \\
\hline Fatigue & 64 & 0 & 96 & $21-24$ & 0.0024 & 0.1368 \\
\hline Fever & 91 & 9 & 81 & 18 & 0.6901 & 0.6992 \\
\hline Myalgia & 55 & 0 & 75 & $4-17$ & 0.1631 & 0.6119 \\
\hline Nausea & 45 & 9 & 66 & $5-9$ & 0.2014 & 0.5549 \\
\hline Vomiting & 18 & 0 & 66 & 5 & 0.0027 & 1.0000 \\
\hline Myelosuppression & 100 & 64 & 92 & $26-60$ & 1. 0000 & 0.2233 \\
\hline Elevated AST/ALT & 91 & 9 & 63 & $14-29$ & 0.0980 & 0.4717 \\
\hline Depression/neuropsychiatric & 46 & 9 & 40 & $2-10$ & 0.7567 & 0.5030 \\
\hline Dose modification & \multicolumn{2}{|c|}{9} & \multicolumn{2}{|c|}{50} & \multicolumn{2}{|c|}{0.0101} \\
\hline
\end{tabular}

Two-sided $P$-values $\leq 0.05$ are considered statistically significant.

Adult toxicity profile data taken from Kirkwood et al., 2002 [32]. When ranges were reported, the midpoint was used for statistical comparisons. 
color of a lesion and the earliest symptom, although uncommon, is persistent itch of a lesion [37]. Ulceration and bleeding are typically associated with deep melanomas at time of diagnosis [37]. Delay in diagnosis was either due to procrastination in presentation by patients and families to their doctor and/or initial clinical misdiagnosis and hesitation in obtaining a biopsy by a health care professional. Melanoma recognition in children can be especially difficult for several reasons [5]. First, because the diagnosis is uncommon in children, melanoma is rarely suspected. Second, the clinical characteristics are often different from the characteristics seen in adults. In adults, melanomas are often asymmetrical, with irregular borders, color variation, and size greater than $6 \mathrm{~mm}$ in diameter. However, in children, particularly those under 12 years of age, melanoma is frequently amelanotic and without particularly worrisome features $[5,38]$. Because the clinical differential diagnosis includes benign lesions such as a Spitz nevus, verruca, pyogenic granuloma, and hemangioma, diagnosis may be delayed. Lastly, physicians may be reluctant to perform biopsies in children. Unfortunately delay in diagnosis can lead to more advanced disease at diagnosis and poorer prognosis. Awareness that melanoma can occur in children is critical to early detection. Children develop nevi that grow as children age, but the degree to which a lesion changes should be a primary factor in the decision to biopsy.

Histologic diagnosis of melanoma can be challenging in children [39-41]. In this study, primary lesions diagnosed as atypical epithelioid melanocytic proliferations or atypical Spitz tumor with indeterminate malignant potential were more common than lesions interpreted as unequivocal melanoma. A Spitz nevus is a benign melanocytic lesion that shares histologic features with melanoma [42]. Clinically, Spitz nevus is common in young people and appears as a small smooth dome-shaped pink-tan papule. Generally, the histopathologic diagnosis of Spitz nevus is straightforward but in some cases, distinguishing this lesion from a melanoma with spitzoid features can be difficult. It is now generally accepted that spindle and epithelioid melanocytic proliferations lie in a histologic continuum with benign Spitz nevus at one end and Spitz-like melanoma at the other $[39,43,44]$. A lesion with histologic features of both Spitz nevus and melanoma may be diagnosed as an atypical Spitz tumor with indeterminate malignant potential. In pediatrics, this problem is not uncommon because Spitz nevi occur more often in children than adults. Su et al. reviewed the cases of 18 patients with atypical or borderline spitzoid melanocytic proliferations who underwent SLN biopsy and found that $55 \%$ had positive SLNs [45]. The mean age of patients with lymph node involvement was 14 years. This study and others support the use of SLN biopsy to aid in diagnosis and prognosis when differentiating between an atypical melanocytic proliferation with indeterminate malignant potential and melanoma is difficult if not impossible [45-47]. In eight patients in this study with micrometastases to regional lymph nodes, the primary lesion was interpreted as an atypical epithelioid melanocytic proliferation or atypical Spitz tumor with indeterminate malignant potential.

For patients diagnosed with AJCC stage I melanoma, 5-year survival rates are greater than $95 \%$ [12]. In contrast, patients diagnosed with thick primary melanoma (Breslow depth $>4 \mathrm{~mm}$ ) or regional lymph node metastases (AJCC stage IIB/C or III) have 5-year survival rates between 25 and $70 \%$ [12]. Although surgery is the definitive treatment for melanoma, the increased risk of recurrence and mortality in this subgroup of patients, has justified the development and use of adjuvant therapies in this group. Currently, HDI is the only adjuvant treatment which is FDA-approved for the treatment of high-risk resected melanoma in adults because of increase in relapse-free survival and possibly overall survival. The seminal study that demonstrated efficacy of HDI against melanoma was ECOG trial E1684 [24]. This multicenter trial consisted of 287 patients with high-risk resected melanoma who were randomized to observation or treatment with HDI. At a median follow-up of 7 years, analysis of treatment impact showed favorable outcome for patients treated with HDI. The 5-year relapse-free survival was $37 \%$ in the HDI group compared with $26 \%$ in the observation arm. Similarly, the 5 -year overall survival was $46 \%$ in the HDI group while in the observation group the rate was $37 \%$ [24]. Subsequent ECOG trials, E1690 and E1694, showed improvement in relapse-free survival and/or overall survival in patients treated with HDI versus observation or ganglioside vaccine $[25,26]$. These studies in adults have led to the use of HDI in children with high-risk melanoma.

Yet, the year-long treatment with HDI is associated with significant adverse events. According to Kirkwood et al., the majority of adult patients undergoing treatment with HDI experienced some toxicities, and approximately $50 \%$ of patients required delay or dose reduction of IFN therapy for any reason [32]. Based on data from ECOG trials, grade 3 or 4 toxicities secondary to fatigue, fever, myalgia, nausea, vomiting, myelosuppression, increased transaminases, and depression or neuropsychiatric symptoms were not uncommon in the adults (Table II) [24-26]. Additionally, two patients in the first ECOG trial, E1684, died of liver toxicity which was partly attributed to inadequate monitoring and failure to modify IFN doses compounded by preexisting liver disease [24]. These fatal outcomes resulted in implementation of formal dose modification guidelines in the event of serious hematologic $\left(\right.$ ANC $<500$ cells $/ \mathrm{mm}^{3}$ ) or hepatic (AST/ALT $>5 \times$ normal) adverse reactions. The current recommendations include discontinuation of IFN therapy at the first occurrence of severe neutropenia or transaminitis until resolu- 
tion of the serological abnormalities ( $\leq$ grade 1$)$. The medication may then be restarted at $66 \%$ of the goal dose. In the event of a second adverse event, the medication is again withheld until resolution ( $\leq$ grade 1$)$, and then is reinitiated at $33 \%$ of the starting dose. IFN therapy is discontinued if a third serious toxicity occurs [32]. However, trials using lower doses of IFN to treat high-risk melanoma patients have not demonstrated the same benefits as HDI $[25,48,49]$. Thus, current management guidelines are intended to balance safety while delivering maximum tolerated doses.

The toxicity profile of HDI in the pediatric melanoma population in this study is similar to the profile in adults and includes constitutional symptoms, gastrointestinal side effects, myelosuppression, transaminitis, and depression. Statistical comparison of adverse events experienced by adults (in ECOG trials) and children showed no significant differences for many of the categories (Table II). However, there was a statistically significant difference in the number of patients that required IFN therapy modification in the two groups ( $P$-value 0.0101$)$. Only one pediatric patient $(9 \%)$ required IFN therapy modification in our series, while half of the adult patients had a dose delay or reduction. In the adult population, constitutional symptoms including fatigue, fever, and myalgia were of sufficient magnitude to result in discontinuation of therapy [24]. Although pediatric patients experienced similar frequency and severity of constitutional adverse events, these younger patients were able to continue with HDI therapy without dose adjustments. Serological abnormalities were prevalent in pediatric patients. Myelosuppression and increases in liver enzymes were noted in all or nearly all of the patients and were of significant severity in four patients (36\%). The patients were well-appearing in spite of these laboratory findings and no serious infections were documented. In the one patient with grade 3 transaminitis, bilirubin levels remained normal. These severe adverse events of HDI therapy occurred during the last week of the induction phase and IFN therapy was continued without complications in three of four patients, with normalization of the laboratory abnormalities. These findings indicate that the laboratory changes were dose dependent and generally reversible with dose reduction (to maintenance phase dose). Over time, pediatric patients became tolerant to IFN. During the maintenance course there were no grade 3 or 4 toxicities and many adverse symptoms mitigated. Children tolerated HDI well, and in the setting of well-appearing patients without evidence of serious infection or liver disease, the criteria for dose delay, reduction, and/or discontinuation used for adults may be too rigid in pediatric patients.

The management of pediatric patients with melanoma and atypical melanocytic lesions worrisome for melanoma will evolve as additional information is gained from scientific study. Specifically, a randomized prospective clinical trial comparing the relative efficacy of HDI, melanoma vaccines, immunochemotherapy, or surgery alone is needed for young patients at high risk for disease recurrence. Similar to their adult counterparts, children with high-risk resected melanoma may benefit from adjuvant IFN. The results of HDI in adults have logically led to the use of HDI in children with high-risk resected disease. Although the toxicity of HDI therapy can be substantial, this study demonstrates that this population tolerates HDI at least as well, if not better than the adult population.

\section{REFERENCES}

1. National Cancer Institute: Annual cancer statistics review, including cancer trends, 1950-1985. Washington DC: US Dept of Health and Human Services, NIH publication 1988:88-2789.

2. Surveillance, Epidemiology, and End Results (SEER). Cancer statistics review 1973-1999. National Cancer Institute. Accessed October 7, 2002. seer.cancer.gov/csr/1973-1999/overview.pdf

3. Jemal A, Murray T, Samuels A, et al. Cancer statistics. CA Cancer J Clin 2003;53:5-26.

4. Rigel DS, Carucci JA. Malignant melanoma: Prevention, early detection, and treatment in the 21st century. CA Cancer J Clin 2000;50:215-236; quiz 237-240.

5. Ruiz-Maldonado R, del Rosario Barona-Mazuera M, HidalgoGalvan LR, et al. Giant congenital melanocytic nevi, neurocutaneous melanosis and neurological alterations. Dermatology 1997;195: $125-128$.

6. Ceballos PI, Ruiz-Maldonado R, Mihm MC, Jr. Melanoma in children. N Engl J Med 1995;332:656-662.

7. Williams ML, Pennella R. Melanoma, melanocytic nevi, and other melanoma risk factors in children. J Pediatr 1994;124:833-845.

8. Marghoob AA, Schoenbach SP, Kopf AW, et al. Large congenital melanocytic nevi and the risk for the development of malignant melanoma. A prospective study. Arch Dermatol 1996;132:170-175.

9. Reintgen DS, Vollmer R, Seigler HF. Juvenile malignant melanoma. Surg Gynecol Obstet 1989;168:249-253.

10. Chun K, Vazquez M, Sanchez JL. Malignant melanoma in children. Int J Dermatol 1993;32:41-43.

11. Rao BN, Hayes FA, Pratt CB, et al. Malignant melanoma in children: Its management and prognosis. J Pediatr Surg 1990;25: 198-203.

12. Balch CM, Buzaid AC, Soong SJ, et al. Final version of the American Joint Committee on Cancer staging system for cutaneous melanoma. J Clin Oncol 2001;19:3635-3648.

13. American Joint Committee on Cancer (AJCC). Cancer Staging Manual and Handbook, 6th Edition.

14. Veronesi U, Cascinelli N, Adamus J, et al. Thin stage I primary cutaneous malignant melanoma. Comparison of excision with margins of 1 or $3 \mathrm{~cm}$. N Engl J Med 1988;318:1159-1162.

15. Veronesi U, Cascinelli N. Narrow excision (1-cm margin). A safe procedure for thin cutaneous melanoma. Arch Surg 1991;126:438441.

16. Balch CM, Soong SJ, Smith T, et al. Long-term results of a prospective surgical trial comparing $2 \mathrm{~cm}$ vs. $4 \mathrm{~cm}$ excision margins for 740 patients with $1-4 \mathrm{~mm}$ melanomas. Ann Surg Oncol 2001;8:101-108.

17. Heaton KM, Sussman JJ, Gershenwald JE, et al. Surgical margins and prognostic factors in patients with thick $(>4 \mathrm{~mm})$ primary melanoma. Ann Surg Oncol 1998;5:322-328.

18. Morton DL, Wen DR, Wong JH, et al. Technical details of intraoperative lymphatic mapping for early stage melanoma. Arch Surg 1992;127:392-399. 
19. Gershenwald JE, Tseng CH, Thompson W, et al. Improved sentinel lymph node localization in patients with primary melanoma with the use of radiolabeled colloid. Surgery 1998;124:203-210.

20. Gershenwald JE, Thompson W, Mansfield PF, et al. Multiinstitutional melanoma lymphatic mapping experience: The prognostic value of sentinel lymph node status in 612 stage I or II melanoma patients. J Clin Oncol 1999;17:976-983.

21. Cochran AJ, Balda BR, Starz H, et al. The Augsburg Consensus. Techniques of lymphatic mapping, sentinel lymphadenectomy, and completion lymphadenectomy in cutaneous malignancies. Cancer 2000;89:236-241.

22. Sondak VK, Wolfe JA. Adjuvant therapy for melanoma. Curr Opin Oncol 1997;9:189-204.

23. Sondak VK. Adjuvant therapy for melanoma. Cancer J 2001; 7(Suppl 1):S24-S27.

24. Kirkwood JM, Strawderman MH, Ernstoff MS, et al. Interferon alfa-2b adjuvant therapy of high-risk resected cutaneous melanoma: The Eastern Cooperative Oncology Group Trial EST 1684. J Clin Oncol 1996;14:7-17.

25. Kirkwood JM, Ibrahim JG, Sondak VK, et al. High- and low-dose interferon alfa-2b in high-risk melanoma: First analysis of intergroup trial E1690/S9111/C9190. J Clin Oncol 2000;18: 2444-2458.

26. Kirkwood JM, Ibrahim JG, Sosman JA, et al. High-dose interferon alfa-2b significantly prolongs relapse-free and overall survival compared with the GM2-KLH/QS-21 vaccine in patients with resected stage IIB-III melanoma: Results of intergroup trial E1694/ S9512/C509801. J Clin Oncol 2001;19:2370-2380.

27. Philip PA, Flaherty LE. Biochemotherapy for melanoma. Curr Oncol Rep 2000;2:314-321.

28. Thompson LW, Brinckerhoff L, Slingluff CL, Jr. Vaccination for melanoma. Curr Oncol Rep 2000;2:292-299.

29. McMasters KM, Sondak VK, Lotze MT, et al. Recent advances in melanoma staging and therapy. Ann Surg Oncol 1999;6:467-475.

30. INTRON. A interferon alfa-2b, recombinant for injection (package insert). Kenilworth, NJ: Schering Corporation.

31. Cancer Therapy Evaluation Program, Common Toxicity Criteria Version 2.0, National Cancer Institute. Accessed October 7, 2002. http://ctep.cancer.gov/forms/CTCManual_v4_10-4-99.pdf

32. Kirkwood JM, Bender C, Agarwala S, et al. Mechanisms and management of toxicities associated with high-dose interferon alfa-2b therapy. J Clin Oncol 2002;20:3703-3718.

33. Tate PS, Ronan SG, Feucht KA, et al. Melanoma in childhood and adolescence: Clinical and pathological features of 48 cases. J Pediatr Surg 1993;28:217-222.
34. MacKie RM. Incidence, risk factors and prevention of melanoma. Eur J Cancer 1998;34(Suppl 3):S3-S6.

35. Elwood JM, Jopson J. Melanoma and sun exposure: An overview of published studies. Int J Cancer 1997;73:198-203.

36. Katsambas A, Nicolaidou E. Cutaneous malignant melanoma and sun exposure. Recent developments in epidemiology. Arch Dermatol 1996;132:444-450.

37. Schwartz JL, Wang TS, Hamilton TA, et al. Thin primary cutaneous melanomas: Associated detection patterns, lesion characteristics, and patient characteristics. Cancer 2002;95:1562-1568.

38. Handfield-Jones SE, Smith NP. Malignant melanoma in childhood. Br J Dermatol 1996;134:607-616.

39. Barnhill RL, Flotte TJ, Fleischli M, et al. Cutaneous melanoma and atypical Spitz tumors in childhood. Cancer 1995;76:18331845 .

40. Barnhill RL. Childhood melanoma. Semin Diagn Pathol 1998;15: 189-194.

41. Barnhill RL, Argenyi ZB, From L, et al. Atypical Spitz nevi/ tumors: Lack of consensus for diagnosis, discrimination from melanoma, and prediction of outcome. Hum Pathol 1999;30:513520.

42. Spitz S. Melanomas of childhood. Am J Pathol 1948;24:591609.

43. Piepkorn M. On the nature of histologic observations: The case of the Spitz nevus. J Am Acad Dermatol 1995;32:248254.

44. Spatz A, Calonje E, Handfield-Jones S, et al. Spitz tumors in children: A grading system for risk stratification. Arch Dermatol 1999; 135:282-285.

45. Su LD, Fullen DR, Sondak VK, et al. Sentinel lymph node biopsy for patients with problematic spitzoid melanocytic lesions: A report on 18 patients. Cancer 2003;97:499-507.

46. Johnson TM, Chang A, Redman B, et al. Management of melanoma with a multidisciplinary melanoma clinic model. J Am Acad Dermatol 2000;42:820-826.

47. Zuckerman R, Maier JP, Guiney WB, Jr., et al. Pediatric melanoma: Confirming the diagnosis with sentinel node biopsy. Ann Plast Surg 2001;46:394-399.

48. Creagan ET, Dalton RJ, Ahmann DL, et al. Randomized, surgical adjuvant clinical trial of recombinant interferon alfa- $2 \mathrm{a}$ in selected patients with malignant melanoma. J Clin Oncol 1995;13:27762783.

49. Cascinelli N, Santinami M. Excision of primary melanoma should allow primary closure of the wound. Recent Results Cancer Res 1995;139:317-321. 